\title{
Effect of Bilateral Mandibular Osteodistraction on the Condylar Cartilage: An Experimental Study on Rabbits
}

\author{
Reda F. Elgazzar ${ }^{1,3}$, Tarek H. El-Bialy ${ }^{2,3, *}$ and Eman Megahed ${ }^{3}$ \\ ${ }^{I}$ Division of Oral and Maxillofacial Surgery, Faculty of Dentistry, University of Manitoba; ${ }^{2}$ Division of Orthodontics, \\ University of Alberta, Edmonton, Canada and ${ }^{3}$ Faculty of Dentistry, Tanta University, Egypt
}

\begin{abstract}
Although various aspects of bone formation during distraction osteogenesis have been studied extensively, there are only limited experimental data concerning the influence of hyper-physiologic mandibular distraction rate on structural alterations in the temporomandibular joint (TMJ) condylar cartilage. The purpose of this study was to evaluate the effect of bilateral distraction osteogenesis of the mandibular body, at a hyper-physiologic rate and length, on the integrity of the condylar cartilage in rabbits. MATERIALS AND METHODS: Eighteen healthy adult male rabbits weighing 2 to $3 \mathrm{~kg}$ were assigned to 1 of 2 groups: the control group $(\mathrm{n}=2$ rabbits, 4 joints) or the study group ( $\mathrm{n}=16$ rabbits, 32 joints) four rabbits ( 8 joints) in each subgroup according to the post-distraction period (1,2,3 or 4 weeks). In the control group, rabbits received sham surgery (Osteotomy without distraction) and then left to live for 4 weeks under the same condition of the study group then euthanized using intravenous overdose of pentobarbital sodium. In the study group, an extra oral custom-made distracter was employed to achieve bilateral mandibular hyper physiologic distraction (1.5 mm twice daily for 5 days) distraction. All animals were evaluated clinically and histomorphometrically and results analyzed by MINITABE 13.1 statistical package using ANOVA test. RESULTS: Animals underwent distraction showed obvious changes in condylar surface contour related to length of the follow up period, compared to the control; these changes seemed to be partly reversible. The most pronounced observation was the irregularities and resorption in the anterior part of the condylar cartilage and the subcondylar bone. Moreover, at the first two weeks, the area of resorption was invaded by large number of osteoclasts and chronic inflammatory cells which declined later in the 3rd and 4th weeks and replaced with osteoblastic activities. CONSLUSION: These experimental data showed that distraction rate of $3 \mathrm{~mm}$ per day may lead to degenerative or even early arthritic changes in the TMJ condylar cartilage in the 1st and 2nd post-distraction weeks. However, all condyles showed adaptive and remodeling sings in the following 3rd and 4th weeks.
\end{abstract}

\section{INTRODUCTION}

The temporomandibular joint (TMJ) is one of the most complex joints in the body. Its condyle is composed of a fibrous surface layer, a proliferative zone, hypertrophic cartilage, and bone tissue [1]. The mandibular condylar cartilage is a primary growth centre and also has characteristics of secondary cartilage, which responds to mechanical forces [2]. The TMJ undergoes changes and modifications in its shape and role over the course of a person's lifetime [3].

Distraction osteogenesis was first described [4] for long bone lengthening and popularized by Ilizarov [5]. Its application in the craniofacial area was introduced by Snyder [6] for mandibular lengthening in a dog experimental model. Clinical application of mandibular osteodistraction has been described using extraoral and intraoral devices [7] \& [8].

Although mandibular lengthening has become a common technique, changes in the TMJ have not been well documented. Because distraction appliances are rigidly fixed to the body of the mandible, the distraction procedure leads to

*Address correspondence to this author at the Room 4051, Dentistry/ Pharmacy Centre, University of Alberta, Edmonton, AB, Canada, T6G 2N8; Tel: (780) 492-2751; Fax: (780) 492-1624; E-mails: telbialy@ualberta.ca and telbialy@hotmail.com bony separation by force transduction through both mandibular segments. When the mandible and the TMJ are considered as a functional unit, it is obvious that mechanical loading on the osteotomized mandibles will have an effect on the articular cartilage. In other words, the axial forces may create a push-out effect of the TMJ, leading to structural alterations and/or positional changes. This could result in an unfavorable clinical outcome of mandibular distraction.

Stelnicki [9] studied the changes in TMJ following mandibular distraction osteogenesis in the transverse dimension. They reported that transverse distraction has a multitude of non-transient effects on the joint including flattening, erosion and displacement of the condyle.

Harper [10] observed changes in the TMJ of 7 monkeys after osteotomy and bone distraction of the mandibular symphysis. They reported that no significant morphologic changes were observed in 3 of the monkeys. On the other hand, active osteoblastic activity and hypertrophy of the fibrous and cartilaginous zones were observed in the other 4 monkeys during the initial 4 weeks after the operation. They suggested that hypertrophy in the cartilaginous zone occurred as a response to compression, and the cartilaginous zone became thinner with time, suggesting that the TMJ has significant repair ability, and can reverse the initial response. 
Karaharju-Suvanto [11] applied gradual bone distraction in the region of the ascending ramus in 17 sheep and reported that the condylar processes on both the distraction and non-distraction sides were temporarily affected. However, the nature of the pressure-related alterations in the cartilaginous tissue remains a matter of controversy.

Salter [12] postulated that when 2 opposing regions of articular cartilage are placed in continuous contact under mechanical pressure, diffusion of nutrients from the synovial fluid is altered; producing localized degenerative changes in the underlying articular cartilage. Experimental data from investigations in rabbits and sheep have shown changes in TMJ condylar morphology and antero-posterior dimension, surface contour irregularities and the diameter of the articular cartilage was reduced in the main pressure zones $[11,13]$. In contrast, other authors have reported that the thickness of cartilage tissue increased in regions under elevated pressure $[14,15,22]$. The purpose of this study was to evaluate the TMJ condylar changes due to hyper-physiologic (rate of 3 $\mathrm{mm}$ per day) bilateral distraction osteogenesis of the mandibular body in rabbits.

\section{MATERIALS AND METHODS}

Eighteen healthy adult male rabbits weighing 2 to $3 \mathrm{~kg}$ were assigned to 1 of 2 groups: the control group ( $\mathrm{n}=2 \mathrm{rab}-$ bits, 4 joints) or the study group ( $n=16$ rabbits, 32 joints) four rabbits (8 joints) in each subgroup according to the postdistraction period $(1,2,3$ or 4 weeks). In the control group, rabbits received sham surgery (Osteotomy without distraction) and then left to live for 4 weeks under the same condition of the study group.

\section{Surgical Procedures}

\section{Corticotomy and Device Fixation}

General anesthesia was induced by intramuscular injections of ketamine $(50 \mathrm{mg} / \mathrm{kg}$; Ketalar) and xylazine $(10$ $\mathrm{mg} / \mathrm{kg}$; Rompun; Bayer). In addition, $1.0 \mathrm{~mL}$ of local anesthetic (2\% lidocaine/epinephrine 1:100,000) was injected into the surgical site before beginning the operation to control bleeding.

After shaving the skin over the left and right inferior mandibular borders and sterilizing the site with Betadine, two skin incisions were made from the mandibular angle to the anterior region along the right and left inferior mandibular borders. Corticotomy was performed vertically on both sides (buccally and lingually) at the level of the first mandibular premolar using a tapered fissure bur. Caution was taken to preserve the vascular bundle. Two 7-mm selftapping screws were used to fix the two posterior arms of the device distal to the corticotomy site and one common long self-tapping screw was used to fix the anterior distractor arms to the anterior right and left bony segment.

Layer-to-layer suturing of the periosteum and skin was performed using 3-0 Vicryl (Ethicon, a Johnson \& Johnson Co, Somerville, NJ) and black silk. Immediately after the operation, intramuscular injections of Gentamicin $(5 \mathrm{mg} / \mathrm{kg})$ were given daily for 3 days to prevent infection. After a latency period of 4 days, distraction was performed twice daily at a rate of $1.5 \mathrm{~mm}$ (total $3 \mathrm{~mm}$ per day) for 5 days (total elongation was $15 \mathrm{~mm}$ ). Four animals of the study group were euthanized weekly for 4 weeks, starting by the end of the 1st post-distraction week. Histomorphometric analysis was used to study the changes in the condylar cartilage as well as activity of the osteoblasts and osteoclasts cells.

\section{Clinically}

Postoperatively, animals were examined and followed up clinically until the end of the follow-up period of each group with respect to wound healing, progress of distraction, ability to eat and drink, and deviation of the mandible.

\section{Histopathology}

After harvesting the specimens, condylar surface was examined for irregularities and roughness. The TMJ condyle and its surrounding tissues were removed and placed in neutral formalin for 48 hours. Then, all specimens were washed in normal saline, cut sagittally and then decalcified at room temperature for 4 weeks in $10 \%$ EDTA ( $\mathrm{pH} 7.4$ ) containing $1 \%$ paraformaldehyde. Tissue specimens were then washed, dehydrated, and processed for embedding in paraffin wax. Successive 5- $\mu$-thick tissue sections were cut on a microtome (MT 990; Research \& Manufacturing Co, Inc, Tucson, AZ) and then stained with standard Haematoxylin and Eosin $(\mathrm{H}$ and E) and Masson trichrome stains, for histopathologic and histomorphometric investigations.

Using morphometric analyzer, three measures (anterior, middle and posterior areas) of the total thickness of the condylar cartilage were obtained for each section by measuring perpendicularly from the outer surface of the articular cartilage to the beginning of the bony trabeculae. Under high magnification, the thickness of the different cartilaginous cell layers were histomorphometrically measured in micrometers and compared in all weeks of study and the control group.

\section{Statistical Analysis}

Minitab 13.1 statistical package (Division of Engineering Computing Services (C) 2000 - Michigan State University, East Lansing, MI, USA) was employed to analyze the quantitative results obtained. Descriptive Statistics was employed to calculate the Mean, Standard Deviation values, etc. for all groups. Anderson Darling normality test was used to test the data which was found to be parametric; accordingly, One Way ANOVA was used to compare the results of all postdistraction weeks to the respected passive control.

\section{RESULTS}

\section{Clinical Results}

All animals survived the operation and follow-up period. Wound healing was noticed in all animals; there were no signs of infection, dehiscence, or other pathologies.

\section{Histomorphometric Analysis}

Condylar cartilage is composed of 5 distinct histological layers: a fibrous connective tissue layer, a highly cellular reserve layer, a proliferation layer, a hypertrophic cartilage layer, calcified cartilage layer and then the sub-chondral bone. Animals underwent distraction showed obvious changes in condylar surface contour related to length of the follow-up period, these changes seemed to be at least partly 
reversible. Week 1 rabbits showed various signs of degenerative changes involving the articulating cartilage at the 3 zones of study: anterior, middle and posterior as evidenced by the decrease in their thicknesses $3.45,5.73$, and $3.34 \mu \mathrm{m}$ compared to the same areas in the control group (4.5, 9.51 and $5.51 \mu \mathrm{m}$ respectively). The difference was found to be statistically significant $(P=0.000)$ (Tables 1 and 2$)$. These changes included: thinning of the condylar cartilage, reduction in cells' number in each layer, absence or thinning of the fibrous layer in most of the cases (as compared to normal condyles) (Figs. 1 and 2). (a)

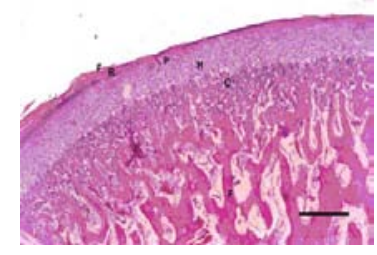

(b)

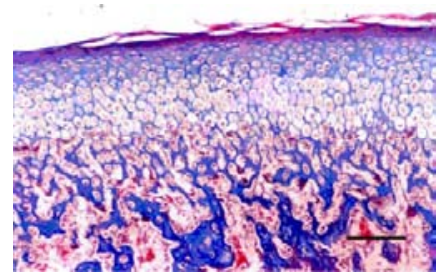

Fig. (1). (a) Normal condylar cartilage consists of well defined regular layers: fibrous $(\mathrm{F})$, reserve $(\mathrm{R})$, proliferative $(\mathrm{P})$, hypertrophic $(\mathrm{H})$, calcified cartilage \& bone $(\mathrm{C})(\mathrm{H} \& \mathrm{E}$ X100) $(\mathrm{Bar}=100$ micrometer). (b) Magnified normal condylar cartilage (Mason trichrome $X$ 200) (Bar = 200 micrometer $)$.

The most pronounced observation was the irregularities and resorption in the anterior part of the condyles including all layers of the condylar cartilage and bone. The area of resorption was invaded by large number of osteoclasts and chronic inflammatory cells. (a)

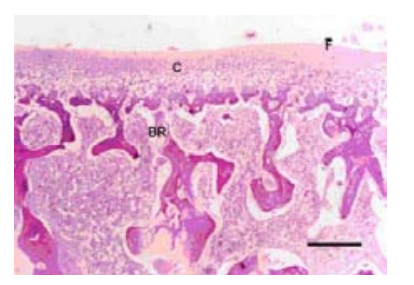

(b)

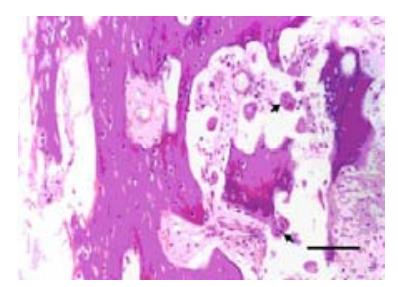

Fig. (2). (a) Photomicrograph of the condyle after 1 week postdistraction showing loss of fibrous layer with reduced thickness of the cartilaginous layer and resorption of the sub-cartilaginous bone (H \& E X100) $($ Bar = 100 micrmemter $)$. (b) Higher magnification showing increased osteoclastic activity (arrows) in the condylar subcartilagenous bone layer (H \& E X200) (Bar = 200 micrometer).

Week 2 rabbits showed some evidence of hypertrophy of all layers of the condylar cartilage (fibrous, proliferative, and hypertrophic layers). A reduction in the number of osteoclasts, a remarkable osteoblastic activity and endochondral ossification were observed during this week (Fig. 3). In week 3 , hypertrophy of the cartilaginous zone was more evident and endochondreal ossification was enhanced and the subcondral zone started to be refilled with new bone trabeculae (Fig. 4). In comparison to the results of the previous weeks, in week 4 , condyles appeared nearly normal; the resorption of the outer margin of the condyle was found to be less than in previous weeks and the cartilaginous layer nearly returned to its pre-distraction (control) thickness (Tables $\mathbf{1}$ and $\mathbf{2}$ and Fig. 5). Furthermore, marked osteoblastic activity was observed whilst the osteoclast cells were not clearly evident in

Table 1. Histomorphometric Measurements (Micrometers) for the Different Histologic Zones of the Condylar Cartilage at 3 Condylar Areas (Anterior, Middle, and Posterior) in 4 Post-Distraction Weeks

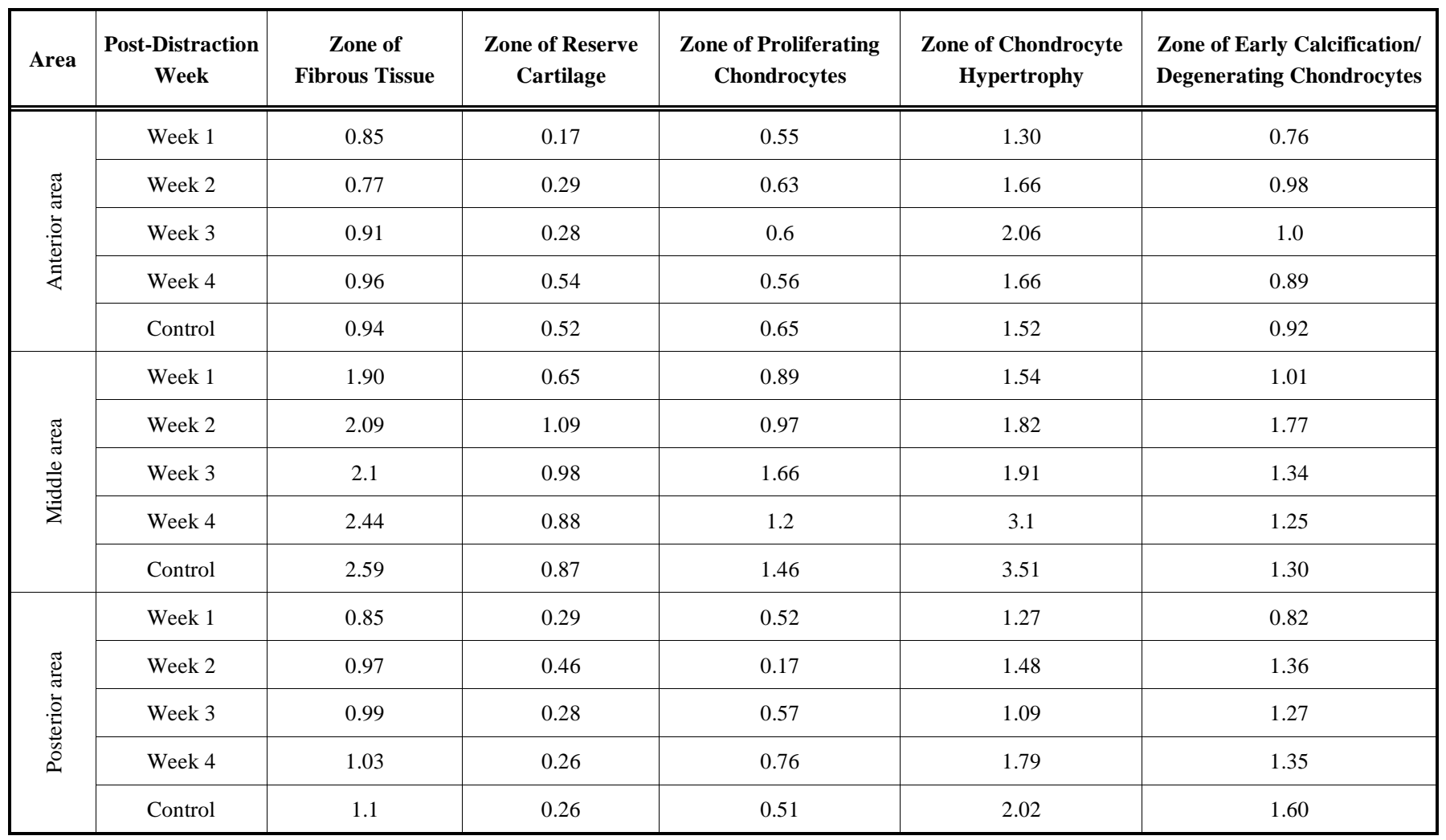


Table 2. Variation in the Thickness (in Micrometer) of the Condylar Cartilage at 3 Condylar Cartilage Areas (Anterior, Middle, and Posterior) in 4 Post-Distraction Weeks (W.) and the Control (Con.) (All the Condylar Histologic Layers are Added Together)

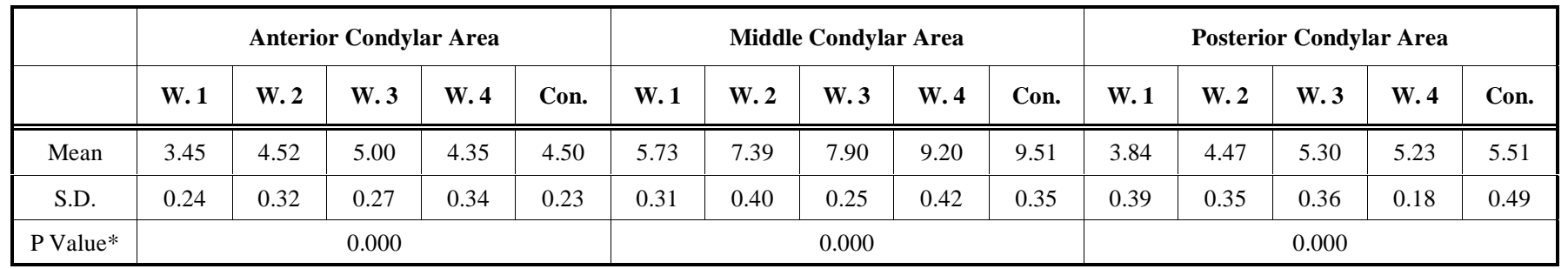

*Using One Way ANOVA.

the bone marrow and this was positively reflected on the density of the condylar bone. (a)

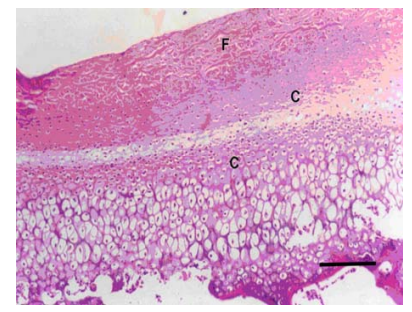

(b)

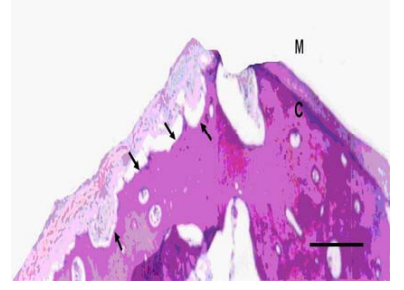

Fig. (3). (a) Microphotograph of the condyle after 2 weeks showing signs of active growth and increased thickness of the fibrous layer and hypercellularity of the chondrocytic layers, which was noticed only in the middle and posterior areas of the condylar surface $(\mathrm{H} \&$ E X100). (b) Microphotograph of the condyle by the end of the 2nd week showing signs of bone resorption and evidence of repair with fibrous tissue, at the anterior articulating area of the condyle $(\mathrm{H} \& \mathrm{E}$ X 40). (a)

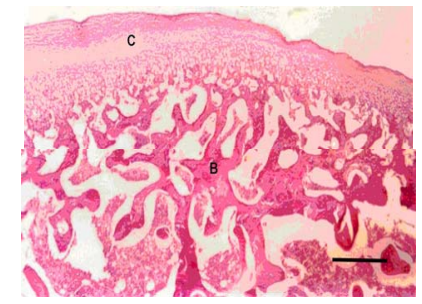

(b)

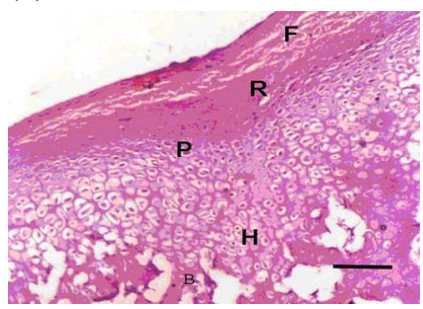

Fig. (4). (a) Microphotograph of the condyle after 3 weeks showing hypertrophy of the cartilaginous layers and reformation of many bone trabeculae (H \& E X100) (Bar = 100 micrometer). (b) Higher magnification of photo no $4 \mathrm{a}(\mathrm{H} \& \mathrm{E}$ X200) (bar $=200$ micrometer). (a)

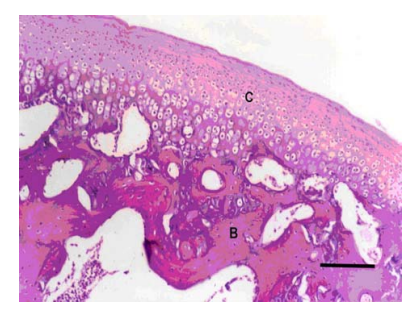

(b)

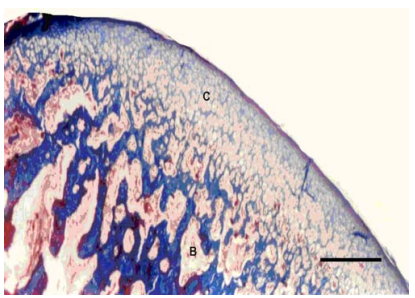

Fig. (5). (a-b). Microphotograph of the condyle after 4 weeks showing histologic features of the anterior part of the condyloid process that looks nearly normal after regeneration of all layers of the articulating cartilage (C) and the subcartilaginous bone (B) ( $\mathrm{a}=\mathrm{H} \&$ E X200, b = Mason Tri-chrome X200) (bar $=200$ micrometer).

\section{DISCUSSION}

Distraction osteogenesis of the mandible is a mainstay in the treatment of craniofacial malformations [8, 16-18]. The risk and the degree of severe malformation in the TMJ after surgical interventions on the mandible have often been reported [18-21]. Despite the high number of investigations focusing on bone regeneration during mandibular distraction, only a few experimental studies have been performed to evaluate the effects of the distraction procedure on the TMJ $[23,24]$. Experimental data from investigations in rabbits and sheep have shown changes in TMJ condylar morphology and antero-posterior dimension, surface contour irregularities and the diameter of the articular cartilage was reduced in the main pressure zones [14, 25, 26]. In contrast, other authors have reported that the thickness of cartilage tissue increased in regions under elevated pressure $[15,27]$.

The current study aimed at evaluating the effect of bilateral distraction osteogenesis of the mandibular body, at a hyper-physiologic rate and length $(1.5 \mathrm{~mm}$ twice a day for 5 days), on the integrity of the condylar cartilage in rabbits at the end of 1st, 2nd, 3rd and 4th post-distraction weeks. In the 1 st and 2nd post-distraction weeks, the results of this study showed prominent signs of degeneration including flattening of the anterior aspect of the condylar head, anterior lipping and contour irregularities as well as reduction in the number and thicknesses of the condylar cartilage layers. Nonetheless, these degenerative changes were partially reduced by remodeling, in most of the cases, in the 3rd and 4th post-distraction weeks. On the other hand, the articular surfaces of the condyle in the control animals were found to be smooth; with no irregularities, erosions or thinning. According to our study, the reaction of the condylar cartilage to the hyper-physiologic distraction force divides into two stages: destructive or degenerative stage (in the 1st and 2nd post-distraction weeks) followed by remodeling or regenerative stage (in the 3rd and 4th post-distraction weeks). Our study highlights the unique regenerative capability of the TMJ in response to the applied distraction force.

Kruse-Lösler [28] studied on rabbits, the effect of distraction osteogenesis on the TMJ condylar cartilage, using various strain magnitudes including: physiologic, elevated and hyper-physiologic magnitudes (2,000, 20,000 and 200,000 microstrains, respectively). Their histological and ultrastructural analyses revealed a positive correlation between the degree of mechanical loading and the development of degenerative alterations in the cartilage. In samples distracted at hyper-physiologic strain magnitudes, all cartilaginous layers were reduced in the regions of the TMJ that had 
been exposed to the higher pressure forces. The fibrous layer became nearly completely destroyed and they concluded that distraction schedules with single but hyper-physiologic loads may lead to degenerative or even early arthritic changes in the condyle. Although this study was carefully done, the follow-up period was carried out for only 2 post-distraction weeks, which represents, according to our study, the destruction or the degenerative stage and this represents only one aspect of the truth. The authors fail to show what happened next in the 3rd and 4th post-distraction weeks which represent, according to our study, the remodeling or regenerative stage and this is the other aspect of the truth.

Kim [29] studied the immunohisto-chemical changes in the TMJ of rabbits in relation to the degree of unilateral mandibular lengthening for $2,3.5$, and $5 \mathrm{~mm}$. They concluded that unilateral mandibular distraction of 2 or $3.5 \mathrm{~mm}$, using a rate of $0.5 \mathrm{~mm} / \mathrm{day}$, was acceptable in that no degenerative changes of the TMJ were observed on either the distraction or non-distraction sides. However, bone resorption was observed in animals that underwent $5 \mathrm{~mm}$ of distraction. Two issues need to be addressed in that study, first is that the follow-up period is only restricted to 2 post-operative weeks and does not show the remodeling phase that may happen after that period; the second is that mandibular lengthening for $5 \mathrm{~mm}$ is not too long in comparison to many other experimental and clinical studies that employed distraction osteogenesis to gain bone length more than $5 \mathrm{~mm}$ without reporting complications in the TMJ.

Secondary cartilage including TMJ appears to respond differently to functional loading than primary cartilage does [30]. Silbermann [31] reported that condylar cartilage contains a population of progenitor cells that synthesize type I rather than type II collagen. Under normal conditions in vivo, local biomechanical factors influence the progenitor cells to differentiate into cartilage cells. These cells are thereby signaled to shift their synthetic pathway from type I collagen to type II collagen that are typical of the cartilage ECM. Nakai [32] conducted a compression test of the TMJ by installing an orthognathic device to perform a crossbite in growing monkeys and found that intermittent compression stimulated endochondral ossification. Copray [33] reported that a continuously applied force of approximately $0.5 \mathrm{~g}$ stimulated the proliferation of condylar cartilage but reduced the synthesis of sulfated glycosaminoglycans and collagen. In contrast to Nakai [32] and to our study, Copray [33] reported that when the condylar cartilage is exposed to intermittent forces, proliferative activity decreases. The apparent contradiction among different studies may be due to variation in the methodology and in the growth stages of the studies animals. Further long term studies are recommended to examine the magnitude of remodeling of the TMJ condylar cartilage along longer post-distraction period and to find out to what extent of mandibular distraction, the TMJ condylar cartilage fails to remodel.

We recommend a follow up study to evaluate TMJ cellular stages as revealed by morphometry using immunostaining as outlined by Luder H.U. [34]. This can be achieved by evaluating proliferating cells nucleic acid (PCNA), apoptotic cells and acid phosphatase activity using immunohistochemistry.

\section{CONCLUSIONS}

In conclusion, our experimental data showed that distraction rate of hyper-physiologic ( $3 \mathrm{~mm}$ per day) may lead to degenerative or even early arthrotic changes in the TMJ condylar cartilage in the 1st and 2nd post-distraction weeks. However, most of the rabbits showed adaptive and remodeling sings in the following 3rd and 4th weeks.

\section{REFERENCES}

[1] Meikle MC. In vivo transplantation of the mandibular joint of the rat: An autoradiographic investigation into cellular changes at the condyle. Arch Oral Biol 1973; 18: 1011-20.

[2] Copray JC, Dibbets JM, Kantomaa T. The role of condylar cartilage in the development of the temporomandibular joint. Angle Orthod 1988; 58: 369-80.

[3] Salo LA, Hoyland J, Ayad S, et al. The expression of types X and VI collagen and fibrillin in rat mandibular condylar cartilage. Response to mastication forces. Acta Odontol Scand 1996; 54: 295302.

[4] Codvilla A. On the means of lengthening in the lower limbs, the muscles and tissues which are shortened through deformity. Am J Orthop Surg 1905; 2: 353-69.

[5] Ilizarov GA. The tension-stress effect on the genesis and growth of tissues. Part I. The influence of stability of fixation and soft-tissue preservation. Clin Orthop Relat Res 1989; 238: 249-81.

[6] Snyder C, Levine G, Swanson H, Browne E. Mndibular lengthening by gradual distraction: Preliminary report. Plast Recons Surg 1973; 51: 506-8.

[7] McCarthy JG. The role of distraction osteogenesis in the reconstruction of the mandible in unilateral cranofacial microsomia. Clin Tech Plast Surg 1994; 21: 625-31.

[8] Sadakah AA, Elgazzar RF, Abdelhady AI. Intraoral distraction osteogenesis for the correction of facial deformities following temporomandibular joint ankylosis: a modified technique. Int $\mathbf{J}$ Oral Maxillofac Surg 2006; 35: 399-406.

[9] Stelnicki EJ, Stucki-McCormick SU, Rowe N, McCarthy JG. Remodelling of the temporomandibular joint following mandibular distraction osteogenesis in the transverse dimension. Plast Reconstr Surg 2001; 107: 647-58.

[10] Harper RP, Bell WH, Hinton RJ, Browne R, Cherkashin AM, Samchukov ML. Reactive changes in the temporomandibular joint after mandibular midline osteodistraction. Br J Oral Maxillofac Surg 1997; 35: 20-25.

[11] Karaharju-Suvanto T, Peltonen J, Laitinen O, Kahri A. The effect of gradual distraction of the mandible on the sheep temporomandibular joint. Int J Oral Maxillofac Surg 1996; 25: 152-6.

[12] Salter RB, Simmonds DF, Malcolm BW, Rumble EJ, MacMichael $\mathrm{D}$, Clements ND. The biological effect of continuous passive motion on the healing of full-thickness defects in articular cartilage. J Bone Joint Surg 1980; 62: 1232-51.

[13] Nakamura E, Mizuta H, Takagi K. Knee cartilage injury after tibial lengthening. Radiographic and histological studies in rabbits after 3-6 months. Acta Orthop Scand 1996; 66: 306-13.

[14] Eggli PS, Hunziker ES, Schenk RK. Quantitation of structural features characterizing weight-and less-weight bearing regions in articular cartilage: A stereological analysis of medial femoral condyles in young adult rabbits. Anat Record 1988; 222: 217-27.

[15] McNamara JA, Hinton RJ, Hoffman DL. Histologic analysis of temporomandibular joint adaptations to protrusive function in young adult rhesus monkeys (Macaca mulatta). Am J Orthod 1982; 82: 288-98.

[16] Pensler JM, Goldberg DP, Lindell B, Carroll NC. Skeletal distraction of the hypoplastic mandible. Ann Plast Surg 1995; 34: 130-36.

[17] Rachmiel A, Levy M, Laufer D. Lengthening of the mandible by distraction osteogenesis: Report of cases. J Oral Maxillofac Surg 1995; 53: 838-46.

[18] Phillips RM, Bell WH. Atrophy of mandibular condyles after sagittal ramus split osteotomy: Report of case. J Oral Surg 1978; 36: 4549.

[19] Weinberg S, Craft J. Unilateral atrophy of the mandibular condyle after closed subcondylar osteotomy for correction of mandibular prognathism. J Oral Surg 1980; 38: 366-68. 
[20] Sesenna I, Faffaini M. Bilateral condylar atrophy after condylar osteotomy for correction of mandibular retrusion. J Maxillofac Surg 1985; 13: 263-66.

[21] Kerstens HCJ, Tuinzing DBH, Golding RP, van der Kwast WA. Condylar atrophy and osteoarthrosis after bimaxillary surgery. Oral Surg 1990; 69: 274-80.

[22] McNamara JA, Carlson DS. Quantitative analysis of the temporomandibular joint adaptations to protrusive function. Am J Orthod 1979; 76: 593-611.

[23] McCormick SU, McCarthy JG, Grayson BH, Staffenberg D, McCormick SA. Effect of mandibular distraction on the temporomandibular joint: Part I, Canine Study. J Craniofac Surg 1995; 6: 35863.

[24] Carlson D. Growth of the temporomandibular joint. In: Zarb GA, Carlsson GE, Sessle BJ, Eds. Temporomandibular Joint and Masticatory Muscle Disorders. Copenhagen, Denmark, Munksgaard, 1994; p 128.

[25] Karaharju-Suvanto T, Peltonen J, Laitinen O, Kahri A. The effect of gradual distraction of the mandible on the sheep temporomandibular joint. Int J Oral Maxillofac Surg 1996; 25: 152-56.

[26] Meyer U, Wiesmann HP, Kruse-Lösler B, Handschel J, Stratmann $\mathrm{U}$, Joos U. Strain-related bone remodeling in distraction osteogenesis of the mandible. Plast Reconstr Surg 1999; 103: 800-07.

[27] Mankin HJ, Brandt KD, Shulman LE. Workshop on etiopathogenesis of osteoarthritis: Proceedings and recommendations. J Rheumatol 1986; 13: 1130 .
[28] Kruse-Lösler B, Meyer U, Flören C, Joos U. Influence of distraction rates on the temporomandibular joint position and cartilage morphology in a rabbit model of mandibular lengthening. J Oral Maxillofac Surg 2001; 59: 1452-59.

[29] Kim SG, Park JC, Kang DW, et al. Correlation of immunohistochemical characteristics of the craniomandibular joint with the degree of mandibular lengthening in rabbits. J Oral Maxillofac Surg 2003; 61: 1189-97.

[30] Ishii M, Suda N, Tengan T, Suzuki S, Kuroda T. Immunohistochemical findings type I and type II collagen in prenatal mouse mandibular condylar cartilage compared with the tibial anlage. Arch Oral Biol 1998; 43: 545-50.

[31] Silbermann M, Reddi AH, Hand AR, Leapman RD, Von der Mark K, Franzen A. Further characterisation of the extracellular matrix in the mandibular condyle in neonatal mice. J Anat 1987; 151: 16988.

[32] Nakai, H, Niimi A, Ueda M. The influence of compressive loading on growth of cartilage of the mandibular condyle in vitro. Arch Oral Biol 1998; 43: 505-15.

[33] Copray JC, Jansen HW, Duterloo HS. Effects of compressive forces on proliferation and matrix synthesis in mandibular condylar cartilage of the rat in vitro. Arch Oral Biol 1985; 30: 299-304.

[34] Luder HU, Leblond CP, von der Mark K. Cellular stages in cartilage formation as revealed by morphometry, radioautography and type II collagen immunostaining of the mandibular condyle from weanling rats. Am J Anat 1988; 182(3): 197-214.

(C) Elgazzar et al.; Licensee Bentham Open.

This is an open access article licensed under the terms of the Creative Commons Attribution Non-Commercial License (http://creativecommons.org/licenses/ by-nc/3.0/) which permits unrestricted, non-commercial use, distribution and reproduction in any medium, provided the work is properly cited. 